\title{
Mean-risk model for portfolio selection with uncertain returns
}

\author{
Wei Li ${ }^{a}$, Weiyi Qian ${ }^{b}$, Mingqiang Yin ${ }^{\mathrm{c}}$ \\ School of Mathematics and Physics, Bohai University, Jinzhou 121000, China \\ awei15241355670@163.com, bqianweiyi2008@163.com, cyinmingqiang2387@sina.com
}

Keywords: Portfolio selection, uncertain measure, uncertain programming, Risk measure.

\begin{abstract}
This paper discusses the uncertain portfolio selection problem when security returns are hard to be well reflected by historical data. In portfolio selection, risk analysis is one of the most important topics and research on quantitative definition of risk remains core of the topic. A new risk measure is introduced in this paper. Based on the new risk function, a mean risk model is proposed. In addition, the gravitation search algorithm is introduced to solve the proposed model. Finally, a numerical example is given to illustrate the modelling idea and the availability of the algorithm.
\end{abstract}

\section{Introduction}

One of the hottest points in applied finance is portfolio selection which is to select a combination of securities among a large number of candidate securities that can best meet the investors' goal. The mean-variance model was proposed by Markowitz [1]. However, this model has limited generality since variance considers high returns as equally undesirable as low returns. Thus, Markowitz [2] proposed semivariance as an improvement measure of risk, and numerous models have been developed based on semivariance.

In the above literatures, security returns are considered as random variables. In many cases, security returns are hard to be well reflected by historical data. Many scholars argued that we should find other theory to solve the portfolio selection problem in this situation. With the introduction of fuzzy set theory and credibility theory, many researches began to employ them to describe and study fuzzy portfolio selection problems. Numerous models containing fuzzy variables are proposed. For example, Bilbao-Terol et al. [3] extended the mean-variance model to fuzzy environment.

However, when fuzzy variables are employed to describe subjective uncertain phenomena, Liu [4] found a paradox. Thus, uncertain measure and further founded uncertainty theory are proposed by Liu [4] which can be used to handle subjective imprecise quantity. Many scholars began to employ the uncertainty theory to describe and study portfolio selection problems. For example, Huang [5] proposed a new risk curve and a new mean-risk model by using the risk free return rate as a prior base target.

In real life, investors usually evaluate the return of a risky investment by comparison to a prior base target they entertain about the investment. But different investors may consider various prior targets. For example, suppose that the risk free return is a benchmark, even if the portfolio return above the risk free return, an investor may still regard it as loss, because the portfolio return has not been exceeded his/her projected target. In this paper, we will also use uncertain variables to describe the experts' estimations of security returns. A new risk measure is discussed and a mean risk model is developed.

The remainder of the paper is organized as follows: some preliminary concepts of uncertainty theory are briefly recalled in Section 2. The new risk measure will be discussed in Section 3. In Section 4, a mean risk model will be proposed. In Section 5, the gravitation search algorithm will be introduced for solving the proposed model. Section 6 gives a numerical example to illustrate application of the new model and the effectiveness of the algorithm. Finally, a brief summary is given in Section 7. 


\section{Necessary knowledge about uncertain theory}

In this section, some basic concepts and theorems are introduced in uncertainty theory.

Let $\Gamma$ be a nonempty set, and $L$ a $\sigma$-algebra over $\Gamma$. Each element of $\Lambda \in L$ is called an event. A set function $M\{\Lambda\}$ is called an uncertain measure if and only if it satisfies the following three axioms:

Axiom 1. (Normality) $M\{\Gamma\}=1$.

Axiom 2. (Self-Duality) $M\{\Lambda\}+M\left\{\Lambda^{C}\right\}=1$ for any event $\Lambda$.

Axiom 3. (Countable subadditivity) For every countable sequence of events $\left\{\Lambda_{i}\right\}$, we have

$M\left\{\bigcup_{i=1}^{\infty} \Lambda_{i}\right\} \leq \sum_{i=1}^{\infty} M\left\{\Lambda_{i}\right\}$

If $M$ is an uncertain measure, then the triplet $(\Gamma, L, M)$ is called an uncertainty space.

In order to define product uncertain measure, Liu [3] proposed the fourth axiom as follow:

Axiom 4. (Product measure) Let $\left(\Gamma_{k}, L_{k}, M_{k}\right)$ be uncertainty spaces for $k=1,2, \cdots, n$. The product uncertain measure is

$M\left\{\prod_{k=1}^{n} \Lambda_{k}\right\}=\min _{1 \leq k \leq n} M_{k}\left\{\Lambda_{k}\right\}$

An uncertain variable $\xi$ can be characterized by an uncertainty distribution which is a function $\Phi: \Re \rightarrow[0,1]$ defined by Liu [3] as $\Phi(x)=M\{\xi \leq x\}$.

Definition 1 [3] Let $\xi$ be an uncertain variable. Then the expected value of $\xi$ is defined by

$E[\xi]=\int_{0}^{+\infty} M\{\xi \geq x\} d x-\int_{-\infty}^{0} M\{\xi \leq x\} d x$,

provided that at least one of the two integrals is finite.

\section{A new risk measure}

For the analogous reasons mentioned in above, we know that different investors may consider different prior targets. Therefore, we define a new risk measure as follows:

Definition 2 Let $\xi$ be an uncertain return of a portfolio, and $T$ be a prior target return for an investor. Then the risk measure is defined by

$R T[\xi]=M\{T-\xi \geq \varphi(T)\}$,

Where $E$ is the expected value operator of the uncertain variable and $\varphi(T)$ is a linear function of T.

Let $\beta$ be the maximum average level that the investors can tolerate. Then it is clear that a portfolio is safe if

$R T[\xi] \leq \beta$.

Theorem 1 Let $\xi$ be an uncertain security return with continuous uncertainty distribution $\Phi(\cdot)$. Then the risk function can be calculated as:

$R T[\xi]=M\{\xi \leq T-\varphi(T)\}=\Phi(T-\varphi(T))$.

\section{Mean risk model and crisp forms}

In this section, the mean risk model will be proposed. 
Suppose there is a $T$ which is a prior target return for an investor. Let $x_{i}$ be the investment proportions in securities $i, \xi_{i}$ the uncertain returns for the $i$ th securities $i=1,2 \cdots, n$. When maximal risk is given, we have the model as follows:

$$
\begin{array}{ll}
\text { Maximum } & E\left[x_{1} \xi_{1}+x_{2} \xi_{2}+\cdots+x_{n} \xi_{n}\right] \\
\text { subject to: } & S T\left[x_{1} \xi_{1}+x_{2} \xi_{2}+\cdots+x_{n} \xi_{n}\right] \leq \beta \\
& x_{1}+x_{2}+\cdots+x_{n}=1 \\
& x_{i} \geq 0, \quad i=1,2 \cdots, n,
\end{array}
$$

Where $\beta$ is the predetermined confidence level accepted by the investor.

Theorem 2. Suppose each security return is the linear uncertain variable denoted by $\xi_{i} \sim L\left(a_{i}, b_{i}\right)(i=1,2, \cdots, n)$. Then the model (5) can be transformed into the following crisp form:

$$
\begin{array}{cl}
\text { Maximum } & \sum_{i=1}^{n}\left(a_{i}+b_{i}\right) x_{i} \\
\text { subject to: } & \left(T-\varphi(T)-\sum_{i=1}^{n} a_{i} x_{i}\right) \leq \beta\left(\sum_{i=1}^{n}\left(b_{i}-a_{i}\right) x_{i}\right) \\
& x_{1}+x_{2}+\cdots+x_{n}=1 \\
& x_{i} \geq 0, \quad i=1,2 \cdots, n,
\end{array}
$$

\section{Gravitation search algorithm}

In order to solve the above nonlinear optimization models, the Gravitation search algorithm (GSA) [5] is introduced. In order to employ the GSA to solve the above constraints optimization model, we use the penalty function method to handle the constraints optimization model. The procedure of the Gravitation search algorithm is summarized as follows:

Step 1. Determine the parameters of the GSA, i.e., the population size N, the initial gravitational constant $G_{0}$, the parameter in the function of gravitational constant $\lambda$.

Step 2. Define the position of the $i$ th agent by $X_{i}=\left(x_{1}^{i}, x_{2}^{i}, \cdots, x_{n}^{i}\right)$ for $i=1,2, \cdots N$.

Step 3. Determine the fitness value function of the agent $i$ at time $t$. The $f i t_{i}(t)$ is defined as follows:

$$
f i t_{i}(t)=-f\left(X_{i}(t)\right)+\gamma \cdot\left[\left\{\max \left\{0, g_{1}\left(X_{i}(t)\right)\right\}\right\}^{2}+\left\{\max \left\{0, g_{2}\left(X_{i}(t)\right)\right\}\right\}^{2}+\left\{h\left(X_{i}(t)\right)\right\}^{2}\right],
$$

Where $f(\cdot), h(\cdot), g_{1}(\cdot)$ and $g_{2}(\cdot)$ are objective function, equality and inequality constraints, respectively, $\gamma$ is a penalty factor.

Step 4. Compute the mass of each agent.

Step 5. Compute the total force that acts on agent $\mathrm{i}$ in a dimension $\mathrm{d}$.

Step 6. Compute the acceleration of the agent $\mathrm{i}$ at time $\mathrm{t}$, and in a direction $\mathrm{d}$.

Step 7. Update the velocity and the position of agent.

Step 8. Repeat the third to the seventh steps for a given number of cycles.

Step 9. Report the best solution.

\section{Numerical example}

In this section, a numerical example is given to illustrate the application of the new model. In addition, the parameters in the GSA are set as follow: population size is set at $60, G_{0}$ is set at $110, \lambda$ is set at 20, and the penalty factor $\gamma$ is set at 1000 .

Example 1 Assume each security return is the Zigzag uncertain variable denoted by $\xi_{i}=Z\left(a_{i}, b_{i}, c_{i}\right), i=1,2, \cdots \cdots 10$. The data set is given in Table 1 . In addition, suppose that the prior target return is 0.1 and the bearable maximum mean loss level is 0.01 . From the model (5), we obtain a crisp optimization model, and employ the GSA to solve this model. In addition, we adjust the $T$ 
value and do the experiment to examine the sensitivity of the base target. The numerical results are given in Table 2.

From Table 2, it is seen that when the prior target return is different, the optimal investment strategy and optimal objective are different, respectively. In addition, by seeing Table 3, it is know that when the tolerable risk level $\beta$ increases, the obtained expected value of the portfolio return becomes greater.

Table 1 Zigzag uncertain return rates of 10 securities.

\begin{tabular}{cccc}
\hline Security $i$ & Uncertain return $\xi_{i}$ & Security $i$ & Uncertain return $\xi_{i}$ \\
\hline 1 & $Z(-0.12,0.26,0.23)$ & 2 & $Z(-0.15,0.28,0.63)$ \\
3 & $Z(-0.62,0.07,0.39)$ & 4 & $Z(-0.02,0.25,0.60)$ \\
5 & $Z(-0.15,0.09,0.74)$ & 6 & $Z(-0.03,0.17,0.53)$ \\
7 & $Z(-0.10,0.15,0.32)$ & 8 & $Z(-0.11,0.01,0.35)$ \\
9 & $Z(-0.34,0.34,0.30)$ & 10 & $Z(-0.21,0.09,0.23)$ \\
\hline
\end{tabular}

Table 2 Investment proportion of 10 securities under different benchmarks (\%)

\begin{tabular}{cllcccccccccc}
\hline$\beta$ & $T$ & Obj. & $x_{1}$ & $x_{2}$ & $x_{3}$ & $x_{4}$ & $x_{5}$ & $x_{6}$ & $x_{7}$ & $x_{8}$ & $x_{9}$ & $x_{10}$ \\
\hline 0.01 & 0.1 & 0.32 & 0.76 & 0 & 0 & 0.42 & 31.53 & 66.62 & 0 & 0 & 0 & 0.37 \\
0.01 & 0.15 & 0.30 & 0.37 & 0 & 0 & 0.2 & 66.26 & 33.11 & 0 & 0.12 & 0 & 0.26 \\
0.01 & 0.2 & 0.27 & 20.51 & 0 & 7.1 & 0 & 67.43 & 6.02 & 0 & 0 & 0 & 0.04 \\
0.01 & 0.25 & 0.24 & 32.49 & 0 & 0 & 0 & 55.36 & 6.9 & 0 & 0 & 0 & 5.25 \\
\hline
\end{tabular}

Table 3 Investment proportion of 10 securities under different risk level constraints (\%)

\begin{tabular}{lcccccccccccc}
\hline$\beta$ & $T$ & Obj. & $x_{1}$ & $x_{2}$ & $x_{3}$ & $x_{4}$ & $x_{5}$ & $x_{6}$ & $x_{7}$ & $x_{8}$ & $x_{9}$ & $x_{10}$ \\
\hline 0.01 & 0.1 & 0.27 & 59.25 & 0 & 0 & 0.23 & 38.35 & 0.53 & 0 & 0 & 2.33 & 0.29 \\
0.015 & 0.1 & 0.36 & 0.37 & 0 & 0 & 0 & 43.26 & 56.11 & 0 & 0.12 & 0 & 0.16 \\
0.02 & 0.1 & 0.47 & 3.51 & 0 & 0 & 25.63 & 12.63 & 67.98 & 0 & 0 & 0 & 0.35 \\
\hline
\end{tabular}

\section{Conclusions}

In this paper, we have discussed the portfolio selection problem when security returns are hard to be well reflected by historical data. Regarding security returns as uncertain variables, the paper has introduced a risk measure and developed a mean risk model. Finally, the gravitation search algorithm was introduced and a numerical example was given to illustrate the effectiveness of the algorithm and the application of the proposed model.

\section{Acknowledgment}

The corresponding author of this paper is Mingqiang Yin. This paper is supported by the National Natural Science Foundation of China (11371071), Scientific Research Foundation of Liaoning Province Educational Department (L2013426).

\section{References}

[1] H.markowitz. Portfolio selection. Journal of Finance. 7 (1952), p.77-91.

[2] H. Markowitz. portfolio selection: Efficient Diversification of Investment. Wiley. New York (1959).

[3] Bilbao-Terol, B.Perez-Gladish, M.Arenas-Parra, et al. Fuzzy compromise programming for portfolio selection. Applied Mathematics and Computation.Vol.173 (2006), p.251-264.

[4] B.Liu. Uncertainty Theory 2nd Edition. Springer-Verlag. Berlin. (2007). 
[5] X. Huang. Mean-risk model for uncertain portfolio selection. Fuzzy Optimization and Decision Making. Vol.10 (2011), p.71-89.

[6] E. Rashedi, H. Nezamabadi-pour, S. Saryazdi. GSA: A gravitational search algorithm. Information Sciences, Vol.179 (2009), p.2232-2248. 